\title{
An Annotation Tool for Semantic Documents (System Description)
}

\author{
Henrik Eriksson \\ Dept. of Computer and Information Science \\ Linköping University \\ SE-581 83 Linköping, Sweden \\ her@ida.liu.se
}

\begin{abstract}
Document annotation is a common technique for relating text and knowledge representation. Although the semantic web emphasizes the annotation of web pages, there are other types of documents that can benefit from ontology-based annotations. PDF documents combined with OWL ontologies form semantic documents that support professional printing, on-line viewing, and ontological models. PDFTab is an extension to the Protégé ontology editor that allows developers to annotate PDF documents with OWL-based ontologies. It is possible to add OWL ontologies to preexisting PDF documents and to relate document parts to concepts in the ontology. PDFTab integrates Adobe Acrobat with Protégé and allows users to switch seamlessly between the document and ontology views. PDFTab illustrates how it is possible to extend semantic-web techniques to the widely-used PDF format while maintaining strong support for ontology development and editing.
\end{abstract}

\section{Introduction}

The semantic web is a well-known approach to adding metadata in terms of ontologies to documents and to facilitating machine-to-machine communication [1]. Although the semantic-web languages and tools are inspired by the web and its underlying formats, such as HTML and XML, there are other possible application frameworks for the semantic-web approach. For example, there is a growing interest in multimedia annotation [2]. Furthermore, there are many types of textual documents that require formats other than HTML. For example, Adobe's Portable Document Format (PDF) is a widely-used electronic-document format, which supports on-line viewing and both desktop and professional printing [3].

The prospect of adding semantic annotations to these document formats is interesting because it makes it possible to extend the scope of the semantic web to include this type of electronic documents to form semantic documents. We have previously explored the benefits of semantic documents based on annotated PDF documents and their potential applications [4, 5]. One conclusion from this work is that, while semantic documents integrate well with regular electronic documents and allow viewing and printing using standard tools, they require new solutions for document annotation. 


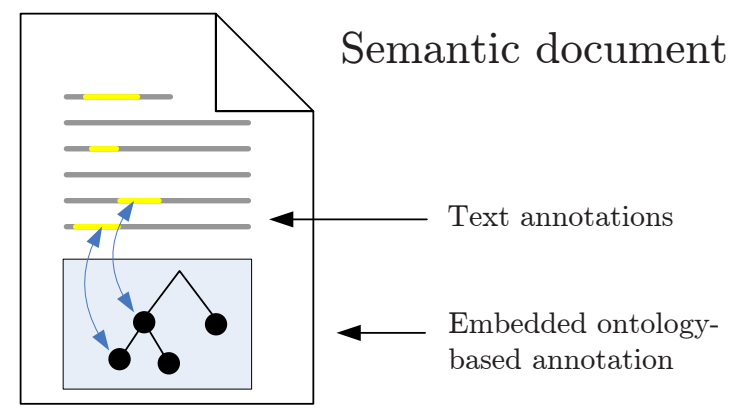

Fig. 1. Semantic documents. The ontology annotations are included in the internal document format.

Adequate tool support is a prerequisite for document annotation. Document formats such as PDF are difficult to create and modify manually (e.g., using a text editor). Thus, we need tools that can both read and write the document format and that can support markup in this format, for instance by selecting document parts and relating them to the ontology. Our approach is to extend the ontology editor Protégé [6] with a plug-in that supports seamless ontology development and annotation of PDF documents. The PDFTab extension allows developers to load PDF documents into Protégé and annotate them in a graphical user interface using Adobe Acrobat. Furthermore, PDFTab allows developers to relate document markup to individuals in the ontology. The advantage of the PDFTab tool architecture is that it retains the rich ontology-editing functionality of the Protégé environment while adding PDF support.

\section{Semantic Documents}

The semantic-document approach combines electronic documents with ontologies 4. The principal idea is to store the ontologies in the internal document representation. Just as RDF and OWL statements can be invisible parts of web pages, semantic documents include ontologies not shown when users view and print the documents. Figure 1 illustrates the semantic-document approach. The documents contain the ontologies and the relationships between concepts in the ontologies and document parts. The rest of the document, including text, graphics, and formatting information, can remain unchanged. This approach allows PDF tools such as Adobe Acrobat to recognize and open the documents in the normal way.

While our approach in general does not assume a particular document format, there are several advantages of using PDF for prototyping and evaluating semantic documents.

1. PDF is a common format for printing and on-line publication. Since the early 1990s, authors and publishers have used PDF for document exchange and publishing. 
2. PDF is an open and documented format [3. There are many commercial and open source applications for generating, modifying, and viewing PDF documents.

3. The PDF specification allows for basic document annotations, such as text highlighting (by color) and the addition of textual notes (similar to MS Word).

4. PDF specifies document compressions and security (e.g, encryption and signing). These methods work for ontologies stored in PDF documents.

In addition, Adobe has added the extensible metadata protocol (XMP) to PDF [7. XMP supports RDF-based metadata in the file formats of several Adobe products. Unfortunately, the way XMP uses the RDF structures in Acrobat makes it incompatible with OWL. Thus, we must store OWL-based metadata for semantic documents separate from XMP in PDF documents.

Compared to HTML, PDF is a much more complex format. One of the major advantages of the HTML format is that it allows document creation and editing with basic tools, such as text editors. Indeed, it is possible to add OWL annotations to HTML documents manually using a text editor (although this requires a lot of work). However, for a complex document format like PDF specialized annotation tools are essential. As mentioned previously, other widely-used formats, such as MS Word, may also be used as the basis for semantic-document markup. Tallis [8] discussed an interesting MS Word extension that supports semantic annotations. Authors can use this tool to add OWL statements to MS Word files interactively.

\section{Annotation Model}

Before presenting the tool architecture, let us first discuss the annotation model. There are several models for adding annotations to documents. In this section, we will discuss three of them. The first possibility is to add metadata to documents without relating the metadata to the document content or parts. XMP, for example, uses this method. The second modeling alternative is to relate the metadata to sections of the document text and other document parts. The advantage of the latter model is that it enables tight integration between documents and ontologies. For example, the model enables users and application programs to use the document text to look up parts of the ontology and vice versa.

Finally, it is possible to store metadata outside the documents, for instance in a separate metalevel database. The advantage of this approach is that no changes are required to the documents. However, the metadata do not follow the documents if they are copied, moved, or communicated to others electronically. Moreover, it is not possible to collect metadata from documents published on the web.

In our work on semantic documents and the PDFTab implementation, we choose the second approach; that is, to store the metadata in the document and to relate document annotations to the ontologies. Our motivation for using this model is that we want to integrate documents and ontologies and keep the metadata within the documents. 


\section{Tool Architecture}

The goal for the tool architecture is to combine advanced ontology development with full support for PDF, such as fully compatible rendering. The strategy is to integrate two state-of-the-art tools to form an environment that supports both perspectives; documents and ontologies. The ontology part of the semanticdocument annotation tool builds on the Protégé environment [6]. The PDF handling and rendering part uses Adobe Acrobat.

Protégé is a suitable basis for the ontology part because it has an advanced graphical user interface and it supports different ontology formats, including OWL 9]. Furthermore, Protégé is a widely-used tool with an active user community. The Java-based Protégé implementation features a core application programming interface (API) for manipulation of ontologies and knowledge bases. The basic system consists of this core API combined with standard plug-ins for graphical editing and storage of ontologies and knowledge bases. This extendability enables us to add new functionality for adding document views to the Protégé user interface and to interconnect Protégé with Adobe Acrobat.

There are many advantages of using Acrobat as the PDF-handling part. Acrobat is the standard tool for creating, manipulating, and viewing PDF documents. There are alternative PDF viewers, but many of these tools may have rendering incompatibilities for certain documents. Specifically, we could not find any Javabased PDF viewers with full PDF support. The main disadvantage of Acrobat, for our purpose, is that there is no support for communication and interoperability with Java programs. It is a major challenge to integrate Protégé with Acrobat because of the different programming languages and communication platforms used. Nevertheless, we believe that the best semantic-document tool solution can be accomplished by integrating these systems.

Figure 2 shows the overall tool architecture. Both Protégé and Acrobat use collections of standard extensions. The PDFTab extension interconnects Protégé and Acrobat using such plug-ins. PDFTab act both as a graphical plug-in that adds a tab to the main Protégé user interface and as a module for managing the communication with Acrobat. The Acrobat plug-in supports graphical markup of PDF documents and manages the communication with the Protégé plug-in. These plug-ins work in concert to enable the users to move seamlessly between the ontology and document views.

An important aspect of the architecture is support for multiple documents. The Adobe and Protégé extensions handle several documents, each with their own ontology. Protégé uses a main ontology, which can import subontologies. In Protégé, users can select the active ontology for editing. Likewise, Acrobat supports several open documents. PDFTab allows developers to associate a list of documents with the main ontology, much like importing the subontologies contained in the documents. The support for multiple documents enables developers to create an ontology structure consisting of a group of semantic documents, which refers to a common main ontology.

The current PDFTab implementation is a research prototype that allows users to experiment with semantic documents and to evaluate the architecture in 


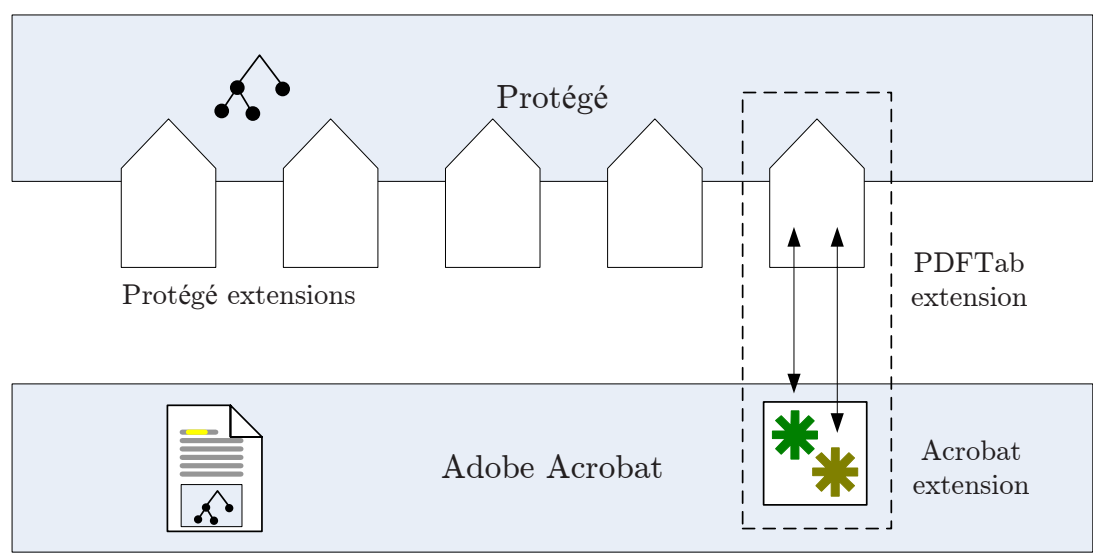

Fig. 2. The PDFTab tool architecture. Extensions of Protégé and Acrobat allow bidirectional communication.

practice. As such, the implementation has a number of limitations. Although Protégé is available on all platforms that support Java and the graphical user interface Swing and Acrobat is available on almost all major platforms (e.g., MS Windows, Apple Macintosh, Sun Solaris, and Linux), the current PDFTab implementation supports the MS Windows platform only. The Adobe plug-in API and inter-application communication protocol is platform dependent, and we choose to focus on the MS Windows platform. The implementation uses the MS Component Object Model (COM) for the communication between the applications, because Acrobat supports this technique.

\section{User Interface}

The overall design goal for the tool user interface is to provide an integrated environment for documents and ontologies. The user interface should follow the general interaction and visual style of both Protégé and Acrobat while making it easy for users to move between the documents and ontologies. Furthermore, the user interface should support handling of multiple documents. Finally, the other Protégé tabs for ontology editing should work as before, including custom and third-part tab extensions.

The user interface of PDFTab consists of two major parts. The Protégé part is a user-interface tab that adds a document view of the Protégé ontology. The Acrobat part consists of additional toolbar buttons for creating three types of semantic annotations and the graphical highlighting of document text and regions. Let us begin by discussing the Protégé document tab. Figure 3 illustrates the layout of the tool user interface with the document tab selected (Fig. 3a). The list of documents provides an overview of the documents associated with the main ontology in Protégé (Fig. 3b). The Acrobat view shows the selected document and allows users to browse it and add annotations (Fig. 3. ). Protégé 


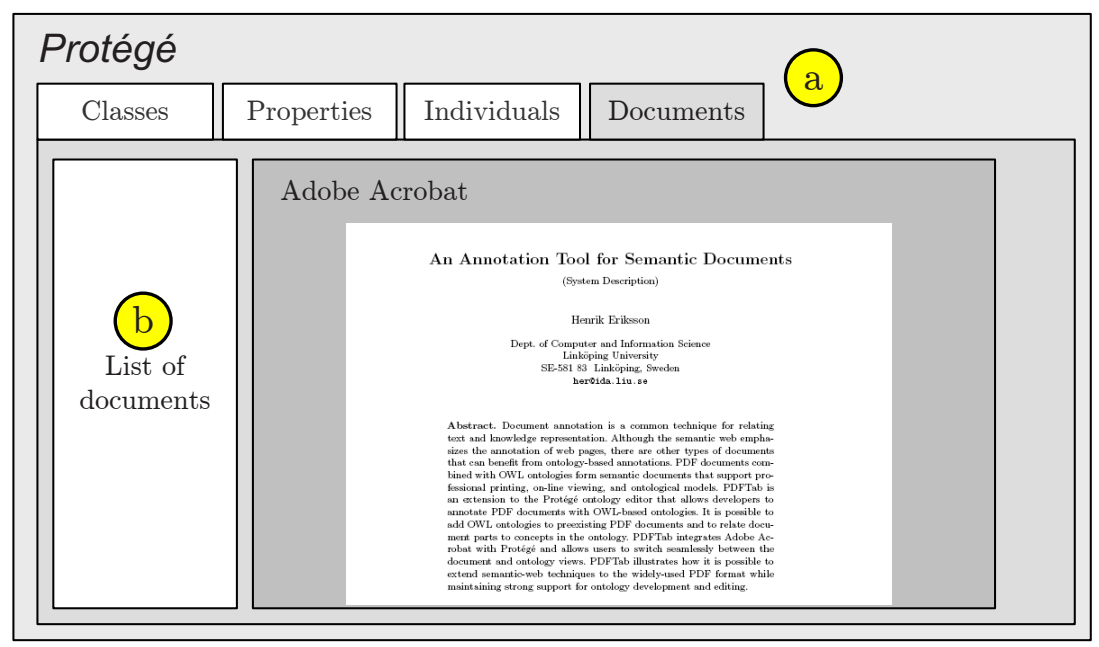

Fig. 3. The organization of the PDFTab user interface. (a) The tab for viewing PDF documents. (b) The list of the documents available. (c) The Acrobat view of the selected document.

uses Acrobat as a user-interface extension just like web browsers, such as MS Internet Explorer and Mozilla Firefox, use Acrobat to support viewing of on-line PDF documents. The main advantage of this user-interface layout is that users can easily move between the document view and one of the ontology views (e.g., classes, properties, and individuals).

\subsection{Document Management}

The support for multiple documents requires user-interface functionality for adding, removing, and inspecting documents. The list of documents (Fig. 3] $\mathrm{b}$ ) contains all documents associated with the project. Users can add existing documents to this list by selecting the document add button or by dragging documents to the list. In PDFTab, the documents must be plain PDF files created by an external application, such as Acrobat Distiller, or PDF-based semantic documents produced by PDFTab. Users can select items from the list of documents for inspecting metadata and for viewing and annotating in the Acrobat view (Fig. 3r). Alternatively, users can access a list of all annotations for the selected document. This annotation browser provides an overview of the document annotations, which is helpful for long documents with many annotations. Selecting an item in the annotation browser brings up the corresponding annotation individual, which is part of an annotation ontology.

\subsection{Annotation Editing}

Users edit annotations in the Acrobat view. PDFTab makes easy to add new annotations to the selected document. To create a new annotation, the user 


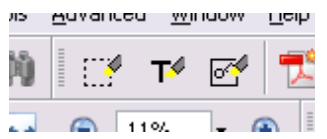

Fig. 4. Annotation buttons in the Acrobat view

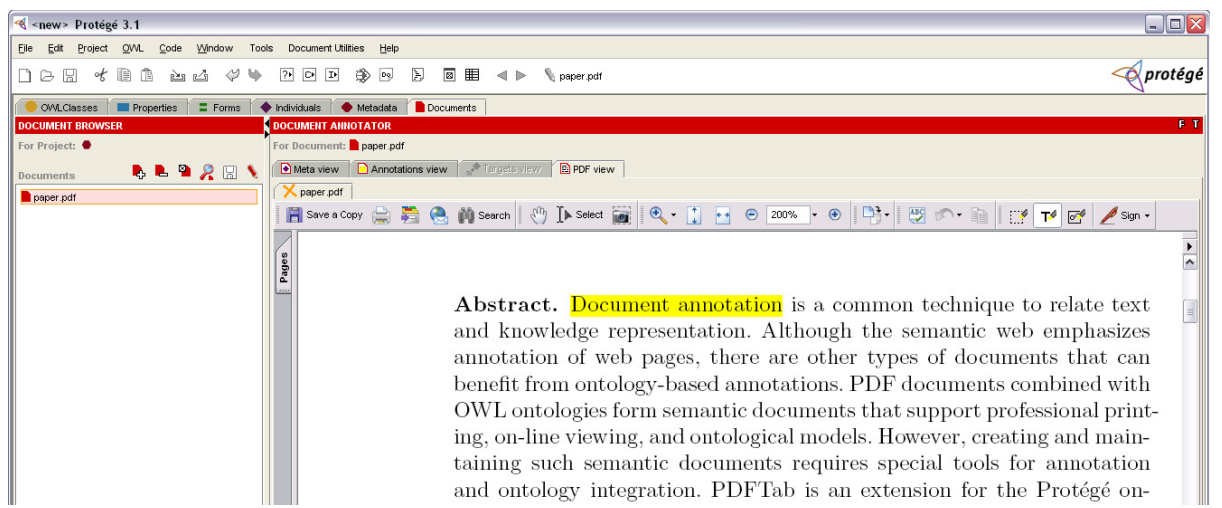

Fig. 5. Document annotation in PDFTab

selects an annotation button, such as the text annotation button, and highlights the text or area to annotate. Figure 4 shows the buttons for the rectangle, text, and graphics annotation tools added to Acrobat. By selecting one of these tool buttons, the user can select a rectangular area, a piece of text, or a figure in the document for semantic annotation. The Acrobat extension highlights the item selected and sends a request to Protégé to prepare the annotation on the Protégé side.

Figure 5 shows the Protégé user interface with the document view, which enables users to browse the document and navigate to the text to annotate. (This screen dump corresponds to the user-interface overview in Fig. 3. Here, the user has selected the text annotation button and highlighted the text "Document annotation". After the user has marked the text, PDFTab creates automatically an OWL individual that represents the annotation, the annotation individual, and opens a form-based editor for this individual (see Fig. 6). In Protégé, users can custom-tailor forms by modifying the layout and selecting widgets for the fields [6]. This functionality enables users to adjust the form for the annotation to suit the subject domain. Furthermore, it is possible to navigate from the document to the ontology by double clicking on the highlighted text, which opens the editor for the annotation individual. Likewise, users can look up annotations in the document by clicking on buttons in the annotation-individual forms.

The annotation individual can refer to classes and individuals in the document's ontology or in another related ontology, such as the main ontology. By default, the annotation class has a target property, which is a pointer to a 


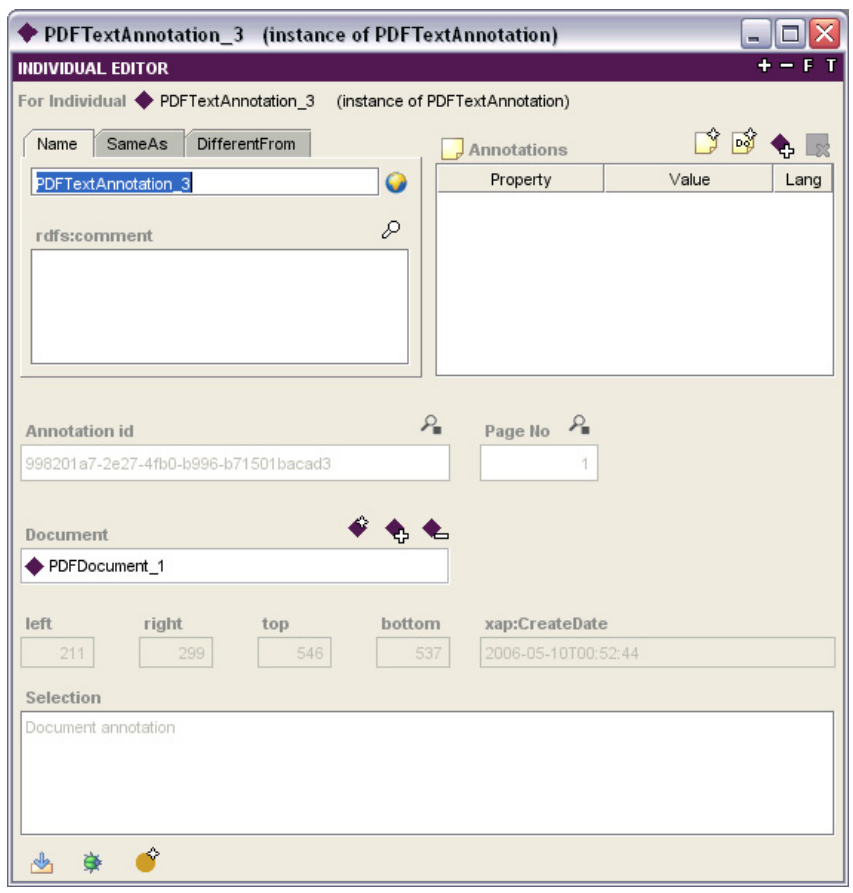

Fig. 6. Form for the annotation individual. This individual corresponds to the annotation in the document. The individual contains information about the annotation id, page number, position, size, creation date, selected text, and an optional reference to a corresponding domain individual.

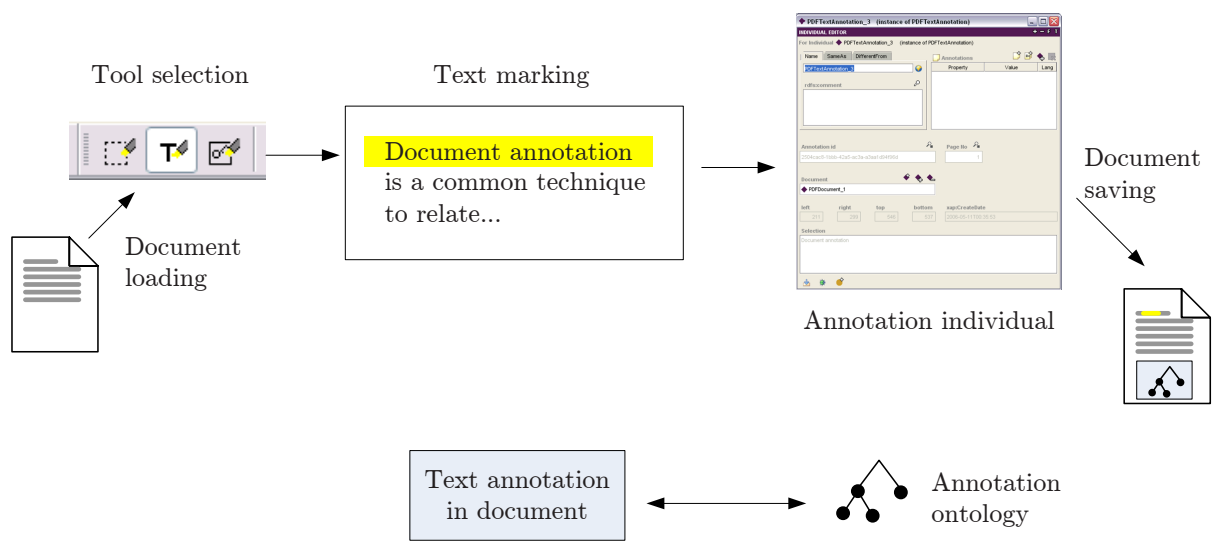

Fig. 7. The annotation process from document loading over annotation creation to document saving 
target individual for the annotation. For example, the target individual can be a class modeling a domain concept or a domain-specific individual. In this case, the annotation individual acts as an intermediate between the document annotation and the domain ontology. This approach helps structuring the ontologies by separating annotation-specific information from domain-specific information.

Figure 7 summarizes the process of creating an annotation. The Protégé and Acrobat extensions load the PDF file (and extracts any previous metadata). Then the user selects the text annotation tool and marks the text to annotate. Next, the Acrobat extension initiates the creation process by highlighting the selected text and sending a creation message to the Protégé extension. Protégé then instantiates the text annotation class to create the annotation individual and opens a form-based editor for the annotation. When the user saves the document, Protégé will serialize all classes and individuals associated with the document and send this structure to Acrobat for inclusion in the document. Acrobat then saves the document as a file.

\section{Discussion}

Integrating documents and ontologies has many advantages. Documents are an important communication and storage format for human knowledge. Ontologies are a structured way of organizing and representing knowledge, especially terminologies and conceptualizations. However, to produce and use semantic documents in practice and extend the vision of the semantic web to documents beyond web pages, it is necessary to have the appropriate infrastructure for handling these documents.

PDFTab is a unique tool in the sense that it supports annotation of PDF documents and combines PDF with advanced ontology-editing support. The user interface combines ontology editing and document viewing in a seamless way and allows users to quickly move between the view, just like using a single application. Furthermore, we believe that the tool architecture is scalable. Both Protégé and Acrobat scale well for large ontologies and documents, respectively. PDFTab already supports multiple documents. Therefore, it is possible to extend the architecture to accommodate for massive document storage in repositories and databases by redesigning the mechanism for handling multiple documents.

The PDFTab approach of combining a standard Windows application with Protégé can serve as an architectural template for other development tools. For example, an interesting prospect is to combine Protégé with other office-oriented applications, such as MS Word and PowerPoint. Such hybrid tools can support new ways of developing ontologies and integrating them with other types of documents and application objects. We believe that the semantic web can benefit from new types of tools that expand the interoperability with common, everyday applications. 


\section{Conclusion}

Extending the semantic web beyond web pages and HTML to complex document and multimedia formats requires new types of tools that can support both ontologies and the target format in an adequate manner. The PDFTab extension of Protégé illustrates the feasibility of efficient support for semantic documents. The combination of Adobe Acrobat and Protégé forms a solid foundation for state-of-the-art handling of documents and ontologies. Although the integration of such different tools requires advanced technical solutions, which adds to the complexity of the system, it is possible to overcome these difficulties and develop hybrid tools that combine the document and ontology views.

\section{Acknowledgments}

This work was supported in part by VINNOVA under grant no. 24478-1 and in part by Statistics Sweden.

\section{References}

[1] Berners-Lee, T., Hendler, J., Lassila, O.: The semantic web. Scientific American 284(5) (2001) 34-43

[2] Stamou, G., van Ossenbruggen, J., Pan, J.Z., Schreiber, G.: Multimedia annotations on the semantic web. IEEE MultiMedia 13(1) (2006) 86-90

[3] Adobe: PDF Reference Version 1.6. 5th edn. Adobe Press, Berkeley, CA (2004)

[4] Eriksson, H.: The semantic document approach to combining documents and ontologies. (in press)

[5] Eriksson, H., Tu, S.W., Musen, M.: Semantic clinical guideline documents. In: Proceedings of the AMIA 2005 Annual Symposium, Washington, DC (October 22-26 2005)

[6] Gennari, J.H., Musen, M.A., Fergerson, R.W., Grosso, W.E., Crubézy, M., Eriksson, H., Noy, N.F., Tu, S.W.: The evolution of Protégé: An environment for knowledge-based systems development. 58(1) (2003) 89-123

[7] Adobe: XMP Specification. Adobe Systems Incorporated (2004)

[8] Tallis, M.: Semantic word processing for content authors. In: Proceedings of the Workshop on Knowledge Markup and Semantic Annotation at the Second International Conference on Knowledge Capture, Sanibel, FL (October 25-23 2003)

[9] Knublauch, H., Fergerson, R.W., Noy, N.F., Musen, M.A.: The Protégé OWL plugin: An open development environment for semantic web applications. In: Proceedings of the Third International Semantic Web Conference, ISWC 2004, Hiroshima, Japan (2004) 229-243 